\title{
XLII. On the mode of obtaining silicium, and on the characters and properties of that substance
}

\section{Berzelius}

To cite this article: M. Berzelius (1825) XLII. On the mode of obtaining silicium, and on the characters and properties of that substance , Philosophical Magazine Series 1, 65:324, 254-267, DOI: $10.1080 / 14786442508628433$

To link to this article: http://dx.doi.org/10.1080/14786442508628433

Published online: 27 Jul 2009.

Submit your article to this journal $₫$

Џ Article views: 4

Q View related articles $\square$ 
ing, a man was pushed on so as to be forced to go two, three, four, or more steps involuntarily, as it may be called, at each push; or if he forced himself so to move by not exerting his propelling powers, except at every second, third, fourth, \&c. time of taking up his leg from the ground.

The advantage of skaiting, then, is in avoiding much of that exertion which is necessary for moving the limbs among themselves, and which has nothing to do with the propelling force.

The practical corollaries I have to draw from these observations are very curious. I have for many years been anxious to realize them, but circumstances have forbidden me-they are still but theories. I think I could show a mechanician not only that the present mode of using animal power for locomotion is very uneconomical, but that there is a better mode feasible, and that that mode would be equally applicable to machines where steam, \&c. is the moving power.

Z. A.

XLII. On the Mode of obtaining Silicium, and on the Characters and Properties of that Substance. By M. Benzeuius*.

Decomposition of Fluate of Silica by Potassium.

WHEN we read the description of the experiments made by MM. Gay-Lussac and Thenard on the silicated Aluoric acid and potassium, we cannot doubt that they decomposed the acid in the circumstances which they describe. The potassium burns in the gas and condenses it; a brown matter is formed, which, boiled in water and dried, burns in oxygen gas with the evolution of silicated fluoric gas, and leaves as a residuum a white earthy matter. In applying myself to these researches, I looked upon the reduction of the fluoric acid and of the silica as so certain, that $I$ thought it only necessary to examine the composition of the product obtained, in order to exhibit the subject in a clear light. The experiment of MM. Gay-Lussac and Thenard gave me the same results as they obtained, with this difference; that the mass when burnt in exygen gas was not white, and that it had preserved its primitive colour without any remarkable change. I expected to find some fluate of silica and potash, but the concentrated sulphuric acid did not disengage any trace of fluate of silica ; and although carried to ebullition, it did not produce any change in the substance. Nor was the substance attacked by any acid but the fluoric, which separated the si-

* From the Annales de Chimie et de Physique, tom. xxvii. p. 337. 
lica, and left a deep brown matter, insoluble in acids, and which did not burn on exposure to the action of fire. Was this the radical of the fluoric acid, or that of silica, or a combination of both?

To procure this substance in greater quantity I proceeded in the following manner: I introduced into a glass retort of the capacity of about ten cubic inches a small vessel of porcelain on which was a piece of potassium of the size of a large nut. After having rapidly exhausted the retort $I$ let in some silicated fluoric gas, from a reservoir over mercury, and I applied the heat of a spirit-lamp. The potassium at first became white, but afterwards it became browner and browner, and at last as black as a coal: it was not long in becoming inflamed, and it burnt with a large flame of a deep-red colour, not however intensely; whilst the mercury rose rapidly in the reservoir; on account of the absorption of the silicated fluoric gas. As soon as the combustion ended, a vacuum was made in the apparatus, in order to prevent the formation of the fluate of silica and potash, and it was then left to cool. The product was a hard, porous, agglutinated, and deep-brown mass, which did not change in the air, but which, like manganese, emitted the smell of hydrogen when it was pressed between the fingers or when breathed upon. In the retort, around the porcelain vessel, $\mathfrak{a}$ light powder of a yellow-brown was remarked, which was preserved. The burnt mass when thrown into water immediately produced a copious evolution of hydrogen gas. The water dissolved much fluate of potash, and the brown mass was precipitated in the form of a chestnut-brown powder, slowly evolving gas. The alkaline solution was decanted and iteplaced by pure water. The evolution of gas visibly diminished; and the water having again been renewed after some time, it entirely ceased; so that the brown powder did not decompose the water, even at the temperature of ebullition. The solution obtained by boiling it being very acid, it was boiled with fresh portions of water, until no signs of acidity were manifested, and the solution afterwards passed through the filtre. The water was saturated with fluate of silica and potash; and the brown powder was washed as long as the water left the slightest residuum by evaporation. The powder, being dried, was of a chestnut-brown, and evidently contained heterogeneous parts of a brighter colour. The brown matter which was deposited on the glass during the combustion of the potassium was much more homogeneous. Water did not. disengage hydrogen by its action, but quickly became acid. This powder was washed with the same care as the precerling.

Now, for the purpose of determining the change undergone 
by the brown matter during combustion, it was first perfectly dried and heated to a dull red in a current of hydrogen gas, and, after being weighed, exposed in a proper apparatus to a current of oxygen gas. As soon as the air of the vessel seemed to be displaced by oxygen gas, the brown matter was heated with a spirit-lamp: it immediately took fire, and burnt vividly for some time, having a pale blue flame on its surface. The remaining oxygen being passed into barytic water, rendered it very turbid, and gave a precipitate which entirely dissolved with effervescence in muriatic acid, and which, consequently, was carbonate of barytes. The burnt mass was greatly diminished in bulk, but was of much the same colour as before. Its weight was augmented scarcely half a hundredth part. There was not perceived either in the glass balloon where the combustion took place, or in the glass conducting tube, the least indication of the presence of fluoric acid; and this showed plainly that this acid was not produced by the combustion of the brown matter; and that the presence of it in the experiments of MM. Gay-Lussac and Thenard, as also in the first which I made, arose from the circumstance that the brown matter contained fluate of silica and potash, which was decomposed by the heat of the combustion, and gave out silicated fluoric acid. This result likewise deprives us of the hope of learning by these means the composition of fluoric acid; but it is not less interesting, since it seems to show that the pulverulent brown matter is really silicium. Its combustion in oxygen, with the production of carbonic acid, without much increase of weight, was now not so difficult to understand, since it is a property of the oxides which contain three atoms of oxygen, that their quadri-carburet burns without increase of weight. But whence comes this carbon? how is it combined with the silicium? I thought at first that it might be attributed to the presence of the oil of naphtha in which the potassium was preserved, and I repeated the experiment with potassium which had not been in this oil. The result was the same. I then began to think that this metal might contain carbon; for it had been prepared after the advantageous process lately given by Brunner, which consists in distilling at a high temperature a mixture of carbonate of potash and of carbon in a vessel of forged iron. The potassium, distilled in a glass vessel, accordingly left a coally residuum, which, by treatment with water, yielded some potash and much carbon. In repeating the experiment of the combustion of potassium in silicated fluoric gas [employing the metal thus purified], the powder was not so brown, and when burnt in oxygen acquired an increase of 40 per cent without producing carbonic 
acid. Its colour, however, was much the same as before combustion. But this circumstance will no longer appear astonishing, if we suppose, either that the silicium may take in its combustion a lower degree of oxidation, or that, as happens with boron, the silica which is formed prevents the entire combustion of the silicium. The residuum of the combustion, treated by fluoric acid, gave silicated fluoric gas, and its colour became much darker. Thrown on a filter, well washed and dried, it was pure silicium.

\section{Description of Silicium, and of its Chemical Relations woith other Bodies.}

Obtained in the manner just related, silicium is of a dark nut-brown colour, without the least metallic lustre: When rubbed with steel it does not give any bright streak, and resists the friction as an earthy body does. It is incombustible in atmospheric air and in oxygen; it does not undergo any change in the flame of the blowpipe; and appears to belong to the class of the most infusible bodies. These properties seem opposed to what I have already said of the combustion of silicium, which is easily accomplished when we employ that substance as obtained immediately after its reduction by potassium. This difference in its combustibility is extremely remarkable. It does not depend on an effect attributable to the fluoric acid; for, if before burning the silicium it is treated with fluoric acid, a portion of silica will be extracted fromit, which had been separated from the fluoric acid by the potash produced by the oxidation of the potassium by the air, before the experiment could be made; and the acid dissolves besides, especially by means of heat, a portion of silicium, with evolution of hydrogen gas: The silicium which then remains after filtering and washing inflames and burns with vivacity in air and in oxygen. Nor can this combustibility result from a residuum of potassium; for after combustion neither fluoric acid nor fluate of silica and potash can be extracted from the product: it appears to me to be owing more probably to a portion of hydrogen combined with the silicium ; for, if the silicium be burnt in oxygen, even after having been heated in hydrogen or in a vacuum, water will be formed, but in very small quantity relatively to the great capacity of saturation of the silica. The silicium which is obtained when the brown mass procured with potassium is thrown into water is, after that, a.hydruret of silicium or a siliciuret of hydrogen: the reduced mass is a siliciuret of potassium, which the water decomposes; the potassium is converted into potash and dissolved; the greater part of the hydrogen is disengaged, and the smaller part takes the
Vol. 65. No. 321. April 1825.
$\mathrm{K} \mathrm{k}$
place 
place of the potassium in combining with the silicium. If the hydruretted silicium is put into an open platinum crucible, and slowly heated until redness commences, and then after being covered carried to a white-red heat, the silicium loses its combustibility; and treated with fluoric acid, which now dissolves no more of it, becomes perfectly pure, without sustaining the great loss which takes place when we begin by burning it.When the hydruretted silicium is very quickly brought to a red heat, it takes fire, because the hydrogen cannot burn at a lower temperature without inflaming the silicium at the same time. If the silicium does not burn completely in this case, that circumstance does not arise from the production of an inferior degree of oxidation, but from the access of oxygen being prevented by the silica formed. Besides the loss of hydrogen which the silicium sustains at an elevated temperature, it undergoes a further change; it loses the property of dissolving in fluoric acid, is contracted in bulk, and takes a darker colour. This circumstance has certainly as great an influence in diminishing its combustibility as the loss of its hydrogen. In the state of least condensation in which it is obtained, when just separated from the potassium by means of water, it may be compared, as to its combustibility, to the porous hydrogenated charcoal of lint, which takes fire by the sparks of a steel, but which loses this property after having been exposed to an elevated temperature. The incombustibility of silicium is besides so great, that the small quantity of it which remains on the filters may be found by burning them and treating the ashes with fluoric acid.

Silicium, even when dry, stains and strongly adheres to the glass vessels in which it is kept. When it is treated with fluoric acid, the liquid is covered with a little pellicle, which envelops, as fat oils do, each drop that is taken from it. This pellicle rises on the sides of the vessels so long as they are damp, and then appears, by refraction, of a clearer colour than the silicium which is under the liquid.

Silicium is a non-conductor of electricity. That which has become incombustible by its exposure to a strong heat does not undergo any change, if, whilst red-hot, chlorate of potash is thrown upon it. It is not attacked by saltpetre until the temperature is raised sufficiently to decompose the nitric acid, and the affinity of the alkali begins to act; but at a white heat it is acted upon with great energy by that salt.

With carbonate of potash, silicium burns very easily with a vivid inflammation; carbonic oxide is given out, and the reduced carbon imparts a black colour to the mass. The incandescence is so much the more intense, and the temperature requires 
requires to be so much less elevated in order to determine the action, as less carbonate of potash or of soda is taken. Thus, for example, taking a portion of carbonate equal in bulk to half the silicium, the inflammation takes place much below the temperature of ignition. With greater quantities of the carbonate, the mass becomes distended by the production of the carbonic oxide, takes fire, and burns with a blue flame. If a still greater quantity of carbonate be employed, no signs of combustion can be perceived; the mass does not grow black, and merely gives out carbonic oxide.

From this mode of action of silicium with carbonate of potash arises a very paradoxical phænomenon. If some incombustible silicium is heated with saltpetre to a moderate red, on a leaf of platinum or in a little crucible, no action will be observed; but if there is added a little dry carbonate of soda, so that it may touch the silicium, a detonation will take place, at the expense of the carbonate, in the midst of the saltpetre, and the mass will preserve for some time its black colour.

The cause from which the silicium burns at a low temperature more easily with the carbonate than with saltpetre, is without doubt owing to the affinity of the alkali for silica being necessary to determine the combustion of the silicium, and not being able to manifest itself with the saltpetre except when the temperature is sufficiently elevated for the decomposition of the nitric acid. If the burnt mass continues black some time longer, this arises from the new combination being compact, and protecting the carbon until it enters into fusion.

Silicium detonates with the hydrates of the fixed alkalies, producing a vivid incandescence at a temperature at which they melt, but much below that of ignition. Hydrogen gas is given out, which is seen to burn when the bulk of the mass is not too small. The same phænomena are observed with the hydrate of barytes. Incandescence also takes place with the hydrate of lime; but it is very feeble, and the silicium is oxidated but imperfectly. With the acid fluate of potash it detonates at the fusing temperature of the salt, that is to say, much below a red heat: it is not affected by fused borax.

Silicium heated to a perfect red in the vapour of sulphur inflames and burns, but with less intensity than in oxygen; and the combination even does not take place with the incombustible silicium. The sulphuration is usually as incomplete as the oxidation, and a scorified mass is obtained of a dark gray colour. It sometimes happens, however, particularly when a vacuum is made in the vessels before volatilizing the $\mathrm{K} \mathrm{k} 2$ sulphur; 
sulphur, that the silicium becomes completely sulphuretted, at least in a part of its mass. It then presents an earthy white body, which, thrown into water, instantly dissolves in it with the evolution of sulphuretted hydrogen. The silicium is converted into silica which dissolves in the water; and if this is in small quantity, a solution may be obtained so much concentrated, that it solidifies after a slight evaporation, and it leaves the silica, after the desiccation, in a transparent fissured mass. Silicium imperfectly sulphuretted also decomposes water rapidly, with the disengagement of hydrogen, and solution of the silica in the water; and the silicium which was not combined with the sulphur becomes_separated. In the air the sulphuret of silicium diffuses a very strong smell of sulphuretted hydrogen; and loses in a little time all its sulphar; but in dry air it may be preserved a long time. At a red heat, it contracts and shrivels up, yielding sulphurous acid and silica. This change, however, takes place but slowly; for when kept at a red heat for some moments, it still has the property of decomposing water.

The siliciuret of potassium readily combines with sulphur at a red heat; but if the mass is dissolved in water, there remains much silicium, unless the mass be newly exposed to a white heat, because the silicium then combines with the sulphur at the expense of the potassium previously sulphuretted at a more elevated temperature. This combination is a true double sulphuret; its colour is of a dark brown, almost black. It presents a melted mass which dissolves in water. It is difficult to say whether it dissolves without changing its nature; but since the sulphuret of silicium is decomposed in water, and sulphuretted hydrogen has a great affinity for the sulphyret of potassium, it is very probable that in the solution there exists some silicate of potash with hydro-sulphuret of the same alkali. The combinations of the sulphuret of silicium with the metallic sulphurets, although they exist in the dry way, cannot, it appears, exist in water.

It is certainly a very remarkable fact to see the silica dissolve in so great quantity in water at the moment of its formation, and lose this property by evaporation, so as to become insoluble in acids. This great solubility, shown by the pre ceding experiments, explains the numerous crystallizations of silica in the cavities of druses, which sometimes could not con tain a valume of liquid ever so little greater than the crystal itself. Nevertheless, I do not pretend that the silica had been in solution as in our example; that is to say, that it was the result of the decomposition of the stlphuret of silicium.

I did not succeed in combining phosphorus with silicium 
by bringing it into contact with the latter at a red heat. I did not try any other process.

If silicium is heated in a current of chlorine, it takes fire and continues to burn. If the gas contains atmospheric air, some silica remains in the form of a slight skeleton. Silicium burns equally well in chlorine, whether or not it has lost its combustibility in the air. The product condenses and presents a yellowish liquid when it contains an excess of chlorine, but which is without colour when it is freed from this excess. This liquid is very fluid; it evaporates almost instantaneously when exposed to the air, yielding white vapours, and leaving a residuum of silica. It has a very penetrating smell, which may be compared in some degree to that of cyanogen. Thrown into water, it floats on the top: it generally'dissolves in it, or leaves a little silica. If the quantity of the water is small, a drop, for example, on as much chloride of silicium, this envelops it, and the silica remains in a frothy semi-transparent mass. This liquid is analogous to the combinations of the other electro-negative bodies with chlorine. It reddens litmus paper: it is the second example known of a liquid combination formed by silicium.

At common temperatures potassium has no action on chloride of silicium; but when heated in the vapour of the latter substance it takes fire and produces a compound of potassium and of silicium. The iodide of potassium does not combine with silicium.

Silicium is neither dissolved nor attacked by the sulphuric, nitric, or muriatic acids, and not even by aqua-regia. In its combustible state it is slowly dissolved by fluoric acid with the evolution of hydrogen; but in losing its combustibility it loses also the property of dissolving in this acid. It is, on the contrary, dissolved with rapidity, even in the cold, by a mixture of the nitric and fluoric acids, nitrous gas being given out. Combustible silicium is dissolved by digestion in a solution of caustic potash; but when rendered incombustible, it is no longer attacked by the alkalies in the humid way.

Silicium, when once isolated, combines with the metals with much difficulty. Its remarkable affinity for platinum is known by the experiments of $M$. Boussingault; but it may be heated as often and as long as we cloose in a platinum crucilde, without any combination taking place. But if we endesyour to reduce silicium by potassium in a platinam crucible the silicium penetrates deeply into the platinum wherever this is touched by the potassium. Copper, silver, lead and tin, heated with silioinm by the blowpipe, do not seem changed in their appearance, nor in their ductility; notwithstanding when 
when they are treated with acids they leave a small quantity of silica. The copper, particularly, left a skeleton of the same form as its own. It is here to be remarked that silicium, which alone is not attacked by the acids, is oxidated by them when its combinations with the metals are dissolved. We have, however; a similar example already in rhodium, which, though not attacked per se by aqua-regia, is dissolved by it when alloyed with certain metals.

Titanium, which approaches so near to silicium in its properties, is also insoluble in acids in the metallic state (with the exception of a mixture of the fluoric and nitric acids), whilst it is oxidated and easily dissolved when alloyed with other metals.

Silicium combines with potassium at a high temperature, but without the evolution of a remarkable heat. It affords two combinations : one, with excess of potassium, of a darkgray, and which dissolves completely in water; the other, with less potassium, is obtained by the reduction before stated, or by exposing the first to a very strong heat. It is besides probable that silicium may form with the metals combinations, the proportions of which correspond with those of their silicates.-I reserve these researches for another time.

\section{Preparation of Silicium.}

The combustion of potassium in silicated fluoric gas requires apparatus which we have not always at hand. The double salts, on the contrary, which fluoric acid forms with silica and potash, or soda, afford very advantageous means of preparing silicium. I did not observe any difference in the use of one or the other of these salts. That of soda has however the advantage of containing under the same weight and the same volume a greater quantity of the fluate of silica. This salt is used in the following manner :-It is reduced to a fine powder; and in case the desiccation has made its particles adhere, and also to expel the humidity that may remain, it is exposed to a temperature as high as it can bear without being decomposed, that is to say, much above $100^{\circ}$. It is then put by layers with the potassium into a tube of glass closed at one end, which is placed in such a manner as that all the mass may be heated at the same time. We may, if we choose, melt the potassium and mix it, by means of a rod of iron, with the salt, and then heat the mass over a spirit-lamp. Even before a red heat, the silicium is reduced with a slight hissing and a feeble appearance of heat. No gas is disengaged when the salt has been well dried. The mass is left to cool, and then treated as before described. It ought however to be dissolved 
in a great quantity of water; so that the liquid, which becomes alkaline by the oxidation of the potassium, may be as diluted as possible, and have less tendency to oxidate and dissolve the silicium. It is for this reason that the mass ought not to be treated with hot water, - that the liquid has not lost its alkaline quality by the washing. It is afterwards boiled with water, and washed with hot water until no stain is left by the evaporation of a drop of liquid. For this purpose much time is generally requisite, and much water must be used. The silicium obtained by this process contains hydrogen; but in a smaller quantity, however, and perhaps in the same manner as the common charcoal of wood, which Davy considers as hydrogenated carbon. It contains also silica, which proceeds principally from the potassium before the reduction, being oxidated a little, and then separating a quantity of silica corresponding to the alkali formed: but the alkali which is formed after the reduction, when it dissolves the mass in the water, dissolves a portion of the double salt in excess, without separating the silica from it. This silica ought to be taken away by means of fluoric acid; but as the silicium would dissolve in the-acid, we must begin by rendering it insoluble and incombustible: if it were made to burn in air, a portion of incombustible silicium would indeed be obtained after treatment by fluoric acid; but ordinarily two-thirds of it would be lost by the combustion. 'This loss is prevented by heating nearly to ignition the dried silicium containing hydrogen in an open crucible: this degree of heat is maintained some time, and then it is raised little by little to a perfect red heat. If the silicium become inflamed, the crucible should be covered directly, and the temperature lowered, which will instantly stop the combustion. When the calcination is finished, the silicium is incombustible in air, and is no longer attacked by fluoric acid, if it does not contain any foreign metal,.iron or manganese for example; for in this case the alloy would be entirely dissolved with disengagement of hydrogen. After treatment by the acid, the silicium is washed and dried. It might be thought that this incombustibility is caused by an extremely thin pellicle of silica; but I dried the silicium in a vacuum, and then heated it to redness in the air, and I did not find any change of weight.

The silica may be reduced by heating it with potassium: but it happens either that the combination, rich in potassium, becomes entirely dissolved in the water; or else, when the heat is sufficient to expel the excess of potassium, that the silicate of potash formed melts into a vitreous mass, and envelops the silicium, which thence acquires a clearer colour. Part of 
the silicate may be separated by means of water, but the remainder can only be removed by fluoric acid. The quantity of silicium obtained is extremely small; and this manner of obtaining it merits attention only because it gives the same result as the double salt when treated with potassium; which proves that the evolution of hydrogen that takes place when the mass is treated by water is owing to the potassium, and not to the combustible radical of fluoric acid. It is by the process in question that Davy endeavoured to reduce silica; and he obtained with the mixed silicate of potash only a brown pulverulent matter, which dissolved in the water, giving it a greenish-gray colour. I observed the same colour in the sohution; but it disappears when this becomes clear.

1 passed silicated fluoric gas through a red-hot iron tube filled: with turnings of the same metal, and it was not sensibly absorbed. The turnings when taken out were of a nut-brown colour, similar to that of silicium, and had the taste of fluate of iron, in the places where the heat had had the most intensity. This last having been dissolved by water, a perceptible pellicle of silicium was left on the surface of the iron, but so thin that it was not possible to separate it. We see by this experiment that iron at a sufficiently elevated temperature has an affinity strong enough to decompose the gas; but that the decompesition soon ceases, because the silicium which is deposited at the surface of the metal-prevents its action. By fusing in a covered iron vessel a mixture of very fine iron filings with fluate of silica and potash, the salt was decomposed and converted into double proto-fluate of iron and potash: after having dissolved it in warm water, a combination of iron and of silicium was left. I hoped to be able to separate the iron by means of an acid; but the silicium became oxidated at the same time, although I employed the liquid silicated fluoric acid. In drying this alloy it oxidated more quickly in the air than it dried, and was changed into an ochre of a rusty yellow colour.

\section{Composition of Silica.}

Since silicium can be obtained in a direct manner, it was natural to endeavour to ascertain its oxidation in a direct manner. For this purpose I burnt 100 parts of pure silicium, dried in a vacuum, by heating it with carbonate of soda. The mass, treated by muriatic acid, evaporated to dryness, and strongly heated, was dissolved in water, and left some silica coloured gray by carbon, which, well washed and ignited, became snowwhite, and weighed $203 \cdot 75$. The solution obtained and the waters of the washing were evaporated afresh, and the saline residuum 
residuum heated to redness. Treating this with water, it still gave a little silica, which, after the addition of a drop of ammonia, took at the expiration of some hours a colour inclining to a yellowish-brown: when heated to redness it weighed 1.5 ; it had lost its dark colour, but it was not snow-white. With soda it gave on platinum foil a faint but evident trace of manganese. 100 parts of silicium had consequently absorbed $105 \cdot 25$ of oxygen, and produced $205 \cdot 25$ of silica. The experiment was repeated on a portion of silicium on which fluoric acid had been evaporated, in order to be more certain that all the silica had been removed. 100 parts of sjlicium, previously calcined to redness in the air, gave, by the process described above, 207 of silica; and by evaporation of the waters, 1 part ; or in all, 208 of silica.

According to these two experiments, silica is composed of Silicium . . . 4. 48.72 to $48 \cdot 08$

Oxygen ... 51.28 to $51 \cdot 92$

They both give a greater proportion of oxygen than that which I found, according to the capacity of saturation of silica for saline bases, and which is only $50 \cdot 3$.

But if we return to the analysis of the salts containing fluate of silica, we shall be able to calculate the saturating capacity of silicium according to the results which we have obtained from it.

Of all these double salts, that of barytes is the most suitable. The only uncertainty which could be met with in its analysis arises from its retaining a little humidity which is not disengaged until the moment of its decomposition. But the quantity of it may be determined by melting the salt with oxide of lead; for the acid is retained, and the water only disengaged. 100 parts of the salt lost in this manner 0.85 of moisture. 100 parts of the same salt, weighed at the same time, gave, according to the process described for the analysis of the fluate of silica and barytes, $82 \cdot 933$ of sulphate of barytes, representing $54 \cdot 428$ of barytes. But according to the analysis already known of the double fluate of silica and barytes, the base is combined with three times more fluoric acid than in the neutral fluate. It thence follows that the double salt is formed of

$$
\begin{aligned}
& \text { Barytes . . . . 54.428 } \\
& \text { Fluoric acid . 22:836 } \\
& \text { Silica . . . . . 21·886 } \\
& \text { Moisture . . . 0.850 } \\
& 100.000
\end{aligned}
$$

The 54.428 parts of barytes are saturated by $7 \cdot 612$ of fluoric acid; whence it follows that $15 \cdot 224$ of this acid were Vol. 65. No. 324. April 1825. L l combined 
combined with 21.886 of silica, or that 100 of acid took 14.3 .76 of silica. The fluate of silica is consequently composed of

$$
\begin{array}{lll}
\text { Fluoric acid . . . . . . . . . . 58.024 } & 100 \cdot 00 \\
\text { Silica . . . . } & 143.76
\end{array}
$$

But 100 parts of fluoric acid correspond to $74 \cdot 7194$, of oxygen in each base that it saturates; consequently this quantity of oxygen ought to be contained in the 143.76 of silex which the acid saturates, and the composition of silica should be

$$
\begin{array}{lll}
\text { Silicium } \ldots \ldots \ldots . .48 \cdot 025 & 100 \cdot 00 \\
\text { Oxygen . . . . . } 51.975 & 108 \cdot 22
\end{array}
$$

This proportion comes very near to that obtained by synthesis, but it is difficult to say on which side the greatest errors of observation are to be found. According to what precedes, the weight of the atom of silicium, supposing that the silica contains three atoms of oxygen, would be $277 \cdot 2$, and, according to the best of the two synthetic experiments, $277 \cdot 8$. The other would carry it to 285 ; but this number by all appearance is too great. It exceeds by $1 \frac{2}{3}$ per cent that which had been adopted at first; which is so well suited for the new and most exact analyses of minerals, that if they were calculated according to the determination which we have just given, they would render an excess of silica necessary. But I must remark on this head, that a mineral is rarely found of which silica does not form an essential component, which does not yet contain from $\frac{1}{2}$ to 2 per cent of it; and even more, either in the state of quartz or in that of another siliceous mineral. This circumstance must take place so much the more with the minerals which have silica for one of their elements ; and consequently all that quantity of it which is found above what the calculation gives should be attributed to a siliceous mixture existing in the mineral.

As to the number of atoms of oxygen contained in silica; the new facts do not furnish us with the means of determining this point. The circumstance that the carburetted silicium produces when burned an equal weight of silica, leads us to consider it as a quadricarburet, which by burning would give an oxide at three atoms of oxygen; but as I have not been able to isolate the carburet of silicium, nor to burn it completely, this result, although obtained in several experiments, and with silicium produced by different operations, has not the certitude it ought to have, to be admitted as a proof. It ought notwithstanding to be considered as affording a strong presumption that silica contains three atoms of oxygen, so far as our knowledge respecting the crystalline form of bodies furnishes us with the means of deciding on the number of atoms of which oxides are formed. For the expression of the com. 
position of the silicates by formulæ, it would certainly be more simple to consider silica as formed of an atom of silicium and an atom of oxygen; but it then would be difficult to conceive the existence of the silicates which contain six times the oxygen of the base, as in the apophyllite, -in which an atom of potash would then be combined with 12 atoms of silica.

To conclude: It still remains to be decided to what class of simple bodies silicium belongs. Since it has no lustre, nor the property of conducting electricity in the state in which it has hitherto been obtained, it is evident that it cannot be classed among the metals, and that its properties bring it near to boron and carbon. Some systematical philosophers will in consequence, doubtless; give it the name of silicon, to indicate by its termination the class of combustibles to which it should be referred. But I look upon this denomination as useless; for there is not any true limit between. the metals and the metalloids. Carbon possesses the metallic lustre, and conducts electricity, but it is not considered as a metal; and if silicium could be melted, it then perhaps would have these properties, which it does not possess in a pulverulent state. Uranium, under this last form, can be distinguished but with difficulty by its appearance from silicium; and when crystallized, on the contrary, it has the metallic lustre and is transparent on the thinnest_edges. Columbium and titanium also approach silicium by their chemical properties; and would it not be well to separate them from the metals, in order to unite them to the metalloids, - that is to say, to the non-metallic combustible bodies? I only wish to show, by these remarks, that there is no natural limit between these bodies; and that when their electric relation only is considered as exact, it is quite indifferent whether we place a combustible body among the metals or not.-(Annalen der Physik und Chemie.)

XLIII. A Letter from the Rev. W. KIRBY in explanation of his Remarks upon the Notice, given in the Philosophical Magazine, of Mr. W. S. MacLeaY's Paper on the Tarsi of certain Insects.

To the Editor of the Philosophical Magazine and Journal.

Dear sir,

Webb's Hotel, Piccadilly, April 14, 1825.

I $T$ gives me great pain and concern to learn that some gen1 tlemen have regarded my remarks upon your statement of the object of Mr.W.S. MacLeay's paper "On the Structure of the Tarsus in the Tetramerous and Trimerous Celeoptera of the French Entomologists," in a light very different from what I intended, and as having the air of an attack upon him. I L 12

hasten 